Revista Brasileira de Agricultura Irrigada v.11, no.8, p. 2172- 2182, 2017

ISSN 1982-7679 (On-line)

Fortaleza, CE, INOVAGRI - http://www.inovagri.org.br

DOI: $10.7127 /$ rbai.v11n800706

Protocolo 706.17 - 27/06/2017 Aprovado em 21/09/2017

\title{
CARACTERIZAÇÃO DE SISTEMA DE IRRIGAÇÃO POR ASPERSÃO CONVENCIONAL DIMENSIONADO COM VAZÃO ECONÔMICA E PRÁTICA E DIFERENTES CONFIGURAÇÕES HIDRÁULICAS ${ }^{1}$
}

\author{
Élvis da Silva Alves²; João Carlos Cury Saad ${ }^{3}$; Ana Paula Russo Assumpção Schimidt ${ }^{4}$; \\ Leandro Moscoso Araújo ${ }^{5}$; Maryjane Diniz de Araújo Gomes ${ }^{6}$; Jannaylton Éverton Oliveira \\ Santos $^{7}$
}

\section{RESUMO}

Apesar do advento dos métodos de irrigação com maior uniformidade, a irrigação por aspersão convencional ainda é responsável por grande parte das áreas irrigadas no Brasil em pequenas e médias propriedades rurais. Por essas propriedades serem menores, não despertam o interesse de empresas de médio e grande porte na realização de projetos, resultando regularmente em projetos mal dimensionados. Em outros casos o projeto apresenta dimensionamento satisfatório no que se diz respeito a atender os critérios agronômicos e hidráulicos, no entanto, o manejo da lâmina de irrigação não é tratado com princípio, ocasionando o manejo ineficiente. Desta forma, este trabalho teve como objetivo verificar o efeito de diferentes vazões (prática e econômica) e configurações hidráulicas no manejo da aplicação de lâminas de irrigação e caracterização de sistema de irrigação por aspersão convencional no momento do dimensionamento. O estudo foi realizado tomando como base características físico-hídricas de uma área do Departamento de Engenharia Rural, Fazenda Experimental Lageado, Unesp/Botucatu-SP. As características estudadas nesse trabalho foram: vazão do projeto (econômica ou prática) e configuração do sistema (A ou B). Para realização dos cálculos foram construídas planilhas eletrônicas no Microsoft Excel®. A vazão prática (VP) permitiu um manejo da irrigação mais eficiente. Ao aumentar a vazão econômica tornando-a prática ocorreu elevação do diâmetro da tubulação da linha principal, como também aumento da perda de pressão na linha lateral. A configuração "A" demandou tubulações com diâmetros maiores. Por sua vez, a configuração "B" demandou maior quantidade de tubulação (diâmetros menores).

Palavras-chave: performance, chuva artificial, agricultura irrigada, irrigação pressurizada.

\footnotetext{
${ }^{1}$ Parte da dissertação do primeiro autor

${ }^{2}$ Eng. Agrônomo, Doutorando em Engenharia Agrícola UFV/Viçosa - MG, e-mail: elvistv@ gmail.com

${ }^{3}$ Eng. Agrônomo, Professor UNESP/FCA, Botucatu - SP, e-mail: joaosaad@ fca.unesp.br

${ }^{4}$ Eng. Agrônoma, Doutoranda em Agronomia UNESP/FCA, Botucatu - SP, e-mail: schimidt.ana@gmail.com

${ }^{5}$ Eng. Agrônomo, Doutorando em Agronomia, UNESP/FCA, Botucatu - SP, e-mail: leandro_moscoso@ hotmail.com

${ }^{6}$ Eng. Agrônoma, Doutoranda em Agronomia UNESP/FCA, Botucatu - SP, e-mail: gomes-mary@ hotmail.com

${ }^{7}$ Eng. Agrônomo, Doutorando em Engenharia Agrícola UFV/Viçosa - MG, e-mail: Eng.js@ @otmail.com.br
} 


\title{
CHARACTERIZATION OF IRRIGATION SYSTEM BY CONVENTIONAL ASPERSION SIZED WITH ECONOMIC FLOW AND PRACTICE AND DIFFERENT HYDRAULIC CONFIGURATIONS
}

\begin{abstract}
Despite the advent of more uniform irrigation methods, conventional sprinkler irrigation is still responsible for most of the irrigated areas in Brazil in small and medium-sized rural properties. Because these properties are smaller, they do not arouse the interest of medium and large companies in the realization of projects, resulting in poorly sized projects on a regular basis. In other cases, the project is satisfactory in terms of meeting agronomic and hydraulic criteria, however, the management of the irrigation blade is not treated with principle, causing inefficient management. In this way, this work had as objective to verify the effect of different flows (practical and economic) and hydraulic configurations in the management of the application of irrigation slides and characterization of irrigation system by conventional sprinkler at the moment of the design. The study was carried out based on physical-water characteristics of an area of the Department of Rural Engineering, Experimental Farm Lageado, Unesp / Botucatu-SP. The characteristics studied in this work were: project flow (economic or practical) and system configuration (A or B). In order to perform the calculations, spreadsheets were built in Microsoft Excel ${ }^{\circledR}$. The practical flow (VP) allowed a more efficient irrigation management. By increasing the economic flow making it practical, there was an increase in the pipe diameter of the main line, as well as an increase in pressure loss in the lateral line. Configuration "A" required pipes with larger diameters. In turn, the "B" configuration required a larger quantity of tubing (smaller diameters).
\end{abstract}

Keywords: performance, artificial rainfall, irrigated agriculture, pressurized irrigation.

\section{INTRODUÇÃO}

A irrigação tem como principal objetivo a reposição da água necessária para o desenvolvimento das culturas, quando as chuvas não são suficientes para suprir esse requerimento. A reposição é realizada através da utilização de sistemas de irrigação, os quais são classificados quando a forma que distribuem a água no solo. São eles: superfície, localizada e aspersão.

Mantovani et al. (2009) afirmaram que a irrigação por aspersão é responsável pela maior parte das áreas irrigadas brasileiras, mostrando a importância deste sistema para a agricultura irrigada das áreas agrícolas do Brasil.

Até o ano de 1996 o cenário das propriedades irrigadas por aspersão era na maioria de produtores com áreas entre 20 e 50 ha. Já no decêndio posterior houve redução no tamanho das propriedades que usavam este método, passando a ser encontrados na maioria das vezes em propriedades com áreas entre $2 \mathrm{e}$ 5 ha, reforçando a ideia de que a irrigação por aspersão, em especial a convencional, é adotada por médios e pequenos irrigantes (CENSO AGROPECUÁRIO 1996, 2006).

Dada a grande variabilidade no formato dessas pequenas e médias áreas, o projeto de irrigação por aspersão convencional permite muitas configurações distintas (BERNARDO et al., 2006), e nem sempre se dimensiona corretamente por não levar em consideração estas características. Para Keller e Bliesner (1990), uma das exigências no dimensionamento de qualquer sistema de irrigação é a construção de um mapa topográfico mostrando os desníveis do terreno, bem como obstáculos e limites agrícolas.

Sabendo que são sistemas instalados em pequenas e médias áreas, observa-se uma restrita participação de grandes empresas realizando este tipo de projeto de irrigação. Esses projetos são realizados por empresas menores, que geralmente não levam em 
consideração as diversas particularidades da área, como formato, vazão e declividade. Entretanto, se faz necessário orientar projetistas e usuários, visando a minimização dos custos do sistema de irrigação, para que se tenha o uso racional de energia e água, remetendo ao rendimento máximo da cultura no que se refere ao quesito dimensionamento do sistema (MORENO et al., 2010).

Quando esses critérios não são levados em consideração, normalmente a quantidade de água é aplicada em excesso, pois o produtor teme que a cultura sofra estresse hídrico (TURCO et al., 2009).

Muitas são as características a serem estudadas em um sistema de irrigação para evitar que este seja mal dimensionado e se torne oneroso. A perda de carga é uma das variáveis específicas no momento do dimensionamento, isso porque ela depende de outros fatores, como vazão e diâmetro da tubulação, desnível do terreno e peças complementares utilizadas na tubulação.

Outro quesito muito importante quando se trabalha com a agricultura irrigada é o manejo da irrigação. Nem sempre um sistema hidraulicamente bem dimensionado remete a um sistema de fácil manejo, isso porque o tempo de irrigação determinado nesses projetos às vezes não permitem a conclusão de números exatos de linhas laterais no total de horas disponível para a irrigação.

É notória a ausência de pesquisas discorrendo sobre possíveis soluções em situações de manejo ineficiente. Desta forma, foi proposto neste trabalho como solução modificar a vazão resultante do projeto (antes tida econômica), tornando-a prática (melhor manejo). Isso só é possível com o aumento da vazão obtida no projeto e consequentemente do aspersor.

Objetivou-se com o trabalho verificar o efeito de diferentes vazões (prática e econômica) e configurações hidráulica na caracterização de sistema de irrigação por aspersão convencional no momento do dimensionamento.

\section{MATERIAL E MÉTODOS}

\section{Informações da área do estudo}

O estudo foi realizado tomando como base na maior parte características físicohídricas de uma área localizada no Departamento de Engenharia Rural, Fazenda Experimental Lageado, na Universidade Estadual Paulista Júlio de Mesquita Filho, campus de Botucatu, nas coordenadas geográficas $23^{\circ} 25^{\prime} 16^{\prime \prime} \mathrm{S}$; $48^{\circ} 43^{\prime} 30^{\prime \prime} \mathrm{O}$ e altitude de 770 metros.

A curva de retenção da água no solo foi determinada no laboratório de Água-Solo, do Departamento de Engenharia Rural da UnespFCA. A densidade do solo (ds) foi determinada no laboratório de mecânica do solo, no mesmo departamento. A velocidade de infiltração básica foi obtida de Gonçalves e Moraes (2012).

O estudo foi feito considerando a cultura do feijão (Phaseolus vulgaris). As demais informações se encontram na Tabela 1. O solo da área foi classificado como Nitossolo Vermelho distroférrico, textura argilosa, de acordo com o Sistema Brasileiro de Classificação de Solos (SISTEMA BRASILEIRO DE CLASSIFICAÇÃO DE SOLOS, 2006).

$\mathrm{Na}$ escolha do aspersor optou-se por aquele em que a Intensidade de Aplicação (mm $\mathrm{h}^{-1}$ ) se apresentou menor que a Velocidade de Infiltração Básica da água no solo $\left(\mathrm{mm} \mathrm{h}^{-1}\right)$, evitando assim escoamento superficial. Outro fator levado em consideração foi a pressão, escolhendo sempre o aspersor que proporcionou a vazão requerida com menor pressão de serviço.

Tabela 1. Informações utilizadas no dimensionamento do sistema de irrigação por aspersão convencional.

INFORMAÇÕES VALORES REFERENCIA




\begin{tabular}{|c|c|c|}
\hline $\mathrm{CC}(\%)^{*}$ & 32 & Laboratório Água-Solo \\
\hline $\operatorname{PMP}(\%)^{*}$ & 18 & Laboratório Água-Solo \\
\hline Ds $\left(\mathrm{g} \mathrm{cm}^{-3} /\right)$ & 1,20 & Laboratório Mecânica do Solo \\
\hline $\operatorname{VIB}\left(\mathrm{mm} \mathrm{h}^{-1}\right)$ & 17,88 & Gonçalves e Moraes, (2012) \\
\hline $\mathrm{Z}(\mathrm{cm})$ & 0,30 & Wutke et al., (2000) \\
\hline$f$ & 0,40 & Brito et al., (2015) \\
\hline Kc & 1,24 & Bizari et al., (2009) \\
\hline ETo $\left(\mathrm{mm} \mathrm{dia}^{-1}\right)$ & 4,00 & Pereira/ et al. (2016) \\
\hline Eficiência do sistema de irrigação (\%) & 80 & - - - \\
\hline Área (ha) & 10,00 & -- \\
\hline Largura da faixa irrigada $(\mathrm{m})$ & 500 & -- \\
\hline Comprimento da faixa irrigada (m) & 200 & - - - \\
\hline Espaçamento entre posições (m) & 18 & - - - \\
\hline Espaçamento entre aspersores (m) & 18 & - - - \\
\hline Horas trabalhadas no dia (h) & 10 & - - - \\
\hline
\end{tabular}

CC - Capacidade de campo; PM - ponto de murcha permanente; Ds - Densidade do solo; VIB - Velocidade de infiltração básica; Z Profundidade efetiva do sistema radicular; $f$ - Fator de depleção; Kc - Coeficiente de cultivo; ETo - Evapotranspiração de referência. * Base de volume.

Na Tabela 2 encontram-se as características dos aspersores utilizados, sendo menor vazão para a vazão econômica e maior vazão para vazão prática.

Tabela 2. Características e especificações dos aspersores utilizados, conforme o tipo de vazão.

\begin{tabular}{|c|c|c|c|c|c|c|}
\hline \multirow[t]{2}{*}{ Vazão } & Pressão & Vazão & $\begin{array}{l}\text { Diâmetro de } \\
\text { Alcance }\end{array}$ & Espaçamento & $\begin{array}{c}\text { Intensidade De } \\
\text { Aplicação }\end{array}$ & \multirow[t]{2}{*}{ Fabricante } \\
\hline & (mca) & $\left(m^{3} h^{-1}\right)$ & \multicolumn{2}{|c|}{ (m) } & $\left(\mathrm{mm} \mathrm{h}^{-1}\right)$ & \\
\hline Econômica & 30 & 2,20 & 30,0 & $18 \times 18$ & 6,8 & Fabrimar $^{\circledR}$ \\
\hline Prática & 30 & 3,32 & 31,8 & $18 \times 18$ & 10,3 & Agropolo $^{\circledR}$ \\
\hline
\end{tabular}

\section{Características avaliadas}

As características estudadas nesse trabalho foram: a) configuração do sistema (A ou B) e b) vazão do projeto (econômica ou prática).

Para realização dos cálculos foram construídas planilhas eletrônicas do pacote Microsoft Office Excel ${ }^{\circledR,}$ dimensionando cada sistema com as especificações descritas. Utilizou-se a metodologia proposta por Keller e Bliesner, (1990) para realização dos dimensionamentos.

\section{Configurações da rede hidráulica}

Foram utilizadas as principais configurações encontradas em campo com a irrigação por aspersão convencional. $\mathrm{Na}$ Figura $1 \mathrm{~A}$ tem-se a configuração mais utilizada, a qual adota apenas uma linha principal com uma linha lateral de cada lado. $\mathrm{Na}$ Figura 1B nota-se que a suposta linha principal divide-se em duas, originando quatro linhas laterais operando simultaneamente. 


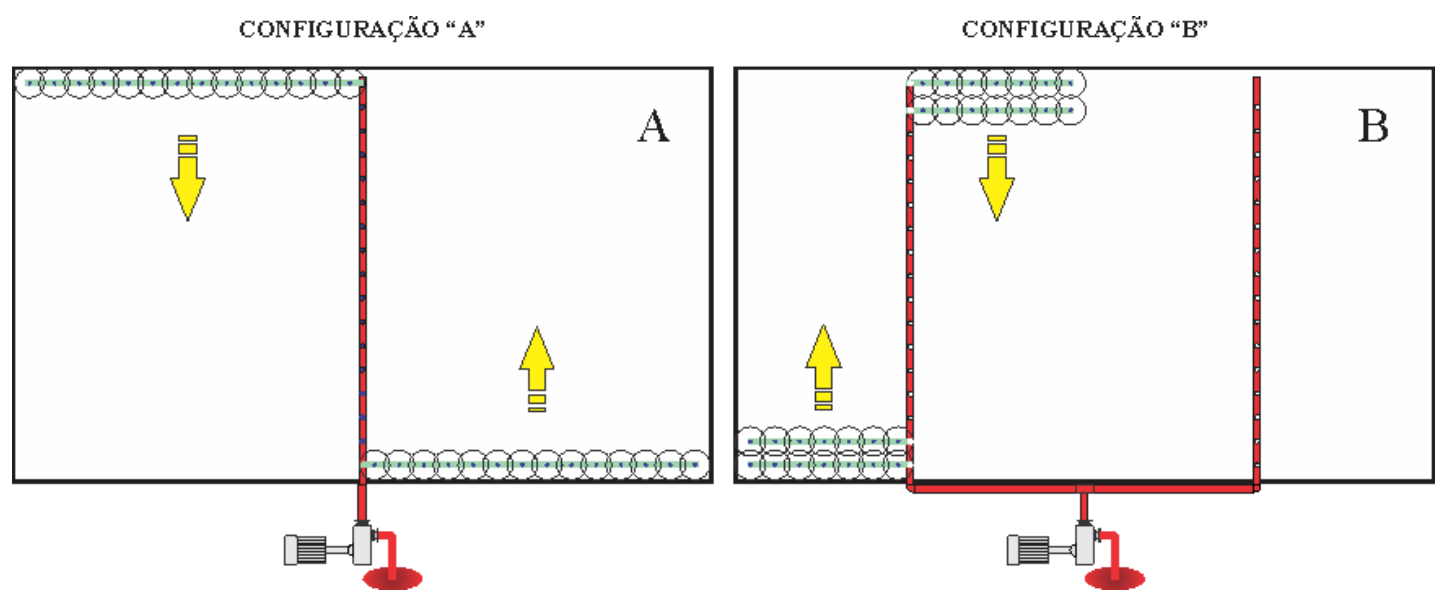

Figura 1. Configurações utilizadas: A) uma linha principal no meio, e; B) duas linhas secundárias ao centro do terreno.

\section{Vazões estudadas}

O sistema foi dimensionado com dois tipos de vazão: "econômica", que maximiza o uso do número de horas disponível para irrigar e utiliza todo o armazenamento disponível no solo para irrigação; e a vazão "prática", que permite ao produtor um manejo com tempo de operação submúltiplo do número de horas disponíveis de trabalho por dia.

\section{RESULTADOS E DISCUSSÃO}

\section{Resultados dos dimensionamentos para as diferentes configurações e vazões}

Na Tabela 3 são encontrados valores referente aos dimensionamentos para todas as situações estudadas (CA, CB, VE; e VP).

Tabela 3. Resultados dos dimensionamentos para as diferentes configurações e vazões

\begin{tabular}{cc}
\hline INFORMAÇÕES & VALORES \\
\hline Disponibilidade Total de Água no solo $\left(\mathrm{mm} \mathrm{cm}^{-1}\right)$ & 1,68 \\
Capacidade Total de Água no solo $(\mathrm{mm})$ & 50,40 \\
Capacidade Real de Água no solo $(\mathrm{mm})$ & 20,16 \\
Irrigação Total Necessária (mm) & 25,20 \\
Turno de rega (dias) & 4,00 \\
\hline
\end{tabular}

\section{Manejo da irrigação utilizando vazão econômica e prática}

As Tabelas 4 e 5 demostram o manejo de irrigação utilizando a vazão econômica e prática, respectivamente. $\mathrm{O}$ dimensionamento eficiente nem sempre permite ao agricultor irrigante um manejo eficiente da sua área, isso porque o tempo de irrigação determinado nesses projetos nem sempre permitem a conclusão de números exatos de linhas laterais no período horário de irrigação, como se observa na Tabela 4.

Tabela 4. Caracterização da aplicação de lâminas de irrigação de acordo com os dados do projeto deste trabalho para configurações A e B.

DIA POSIÇÕES

\begin{tabular}{llllllllllll}
1 & 2 & 3 & 4 & 5 & 6 & 7 & 8 & 9 & 10 & 11 & (horas) \\
\hline
\end{tabular}




\begin{tabular}{ccccccccccccc}
1 & 3,71 & 3,71 & 2,58 & - & - & - & - & - & - & - & - & 10 \\
2 & - & - & 1,13 & 3,71 & 3,71 & 1,45 & - & - & - & - & - & 10 \\
3 & - & - & - & - & - & 2,26 & 3,71 & 3,71 & 0,32 & - & - & 10 \\
4 & - & - & - & - & - & & & & 3,39 & 3,71 & 2,9 & 10 \\
5 & - & - & - & - & - & - & - & - & - & - & 0,81 & 0,81 \\
\hline $\begin{array}{c}\text { Duração por } \\
\text { posição } \\
\text { (horas) }\end{array}$ & 3,71 & 3,71 & 3,71 & 3,71 & 3,71 & 3,71 & 3,71 & 3,71 & 3,71 & 3,71 & 3,71 & Total \\
\hline
\end{tabular}

Na Tabela 4 observa-se que no dia 1 foram irrigadas 2 (duas) posições completas, ficando 1 (uma) incompleta, restando 30,45\% da lâmina de irrigação a ser aplicada no dia posterior. No dia 2 (dois) observou-se uma fragmentação ainda maior da lâmina aplicada nas posições, já que o restante do dia 1 necessitou ser disponibilizado à cultura no dia posterior. Foram aplicados neste dia 30,45; 100; 100 e 39,08\% nas posições 3, 4, 5 e 6, respectivamente. No dia 3 (três) esses percentuais foram de 60,$91 ; 100 ; 100$ e $8,62 \%$, para as posições $6,7,8$ e 9 , respectivamente. Na posição 9 , o percentual de $8,62 \%$ da lâmina de irrigação passará por um intervalo de aproximadamente 14 horas para receber os $91,38 \%$ restante. Por fim, observa-se que no dia 5 ainda foi necessário aplicar o restante da lâmina para posição 11 , já que o turno de irrigação não foi de exatamente 4 dias, havendo um pequeno incremento de tempo. Desta forma, pode-se afirmar que não está havendo a reposição da capacidade de campo, o que ocasiona em estresse hídrico.

A quantidade de água disponibilizada ao solo em forma de irrigação, com objetivo de atender a necessidade da planta, deverá ser o bastante para elevar a umidade deste até a zona efetiva do sistema radicular, elevando o solo até a capacidade de campo (DABACH et al., 2016; SILVA et al., 2000). Para Custódio et al., (2009) o estresse hídrico é um dos fatores que contribuem para a diminuição do rendimento das culturas.

Para solucionar os problemas listados na Tabela 4, propôs-se aumentar a vazão do aspersor e com isso diminuir o tempo de irrigação por linha lateral e consequentemente por posição. Desta forma, surge a "vazão prática", resultado do aumento da "vazão projeto".

Na Tabela 5 exemplifica-se o manejo das lâminas de irrigação já com vazão modificada (aumento de 48,64\%), em que a cada dia quatro posições recebem a lâmina de irrigação no mesmo tempo (2,5 horas).

Tabela 5. Caracterização da aplicação das lâminas de irrigação de acordo com a proposta da vazão prática para configurações A e B.

\begin{tabular}{|c|c|c|c|c|c|c|c|c|c|c|c|c|}
\hline \multirow{2}{*}{ DIA } & \multicolumn{11}{|c|}{ POSIÇÕES } & \multirow{2}{*}{$\begin{array}{r}\text { SOMA } \\
\text { DIÁRIA } \\
\text { (horas) }\end{array}$} \\
\hline & 1 & 2 & 3 & 4 & 5 & 6 & 7 & 8 & 9 & 10 & 11 & \\
\hline 1 & 2,5 & 2,5 & 2,5 & 2,5 & - & - & - & - & - & - & - & 10 \\
\hline 2 & - & - & - & - & 2,5 & 2,5 & 2,5 & 2,5 & - & - & - & 10 \\
\hline 3 & - & - & - & - & - & - & - & - & 2,5 & 2,5 & 2,5 & 7,5 \\
\hline $\begin{array}{c}\text { Duração por } \\
\text { posição } \\
\text { (horas) }\end{array}$ & 2,5 & 2,5 & 2,5 & 2,5 & 2,5 & 2,5 & 2,5 & 2,5 & 2,5 & 2,5 & 2,5 & $\begin{array}{c}\text { Total } \\
(27,50)\end{array}$ \\
\hline
\end{tabular}

$\mathrm{Na}$ situação da Tabela 5, apesar da grande facilidade no manejo tem-se o inconveniente do sistema ficar aproximadamente 1 dia e 7 horas com o sistema parado, já que o aumento da vazão permitiu que a lâmina fosse aplicada mais 
rápido, levando 27,50 horas para finalizar toda a irrigação, sendo que na vazão econômica esse tempo foi de 40,81 horas.

Desta forma, pode-se afirmar que o projeto de irrigação nem sempre deve ser levado em consideração na integra. Diversas particularidades devem ser consideradas, a exemplo da modificação da vazão para o sistema se tornar prático e o manejo ser eficiente

\section{Influência da configuração de implantação da rede hidráulica no dimensionamento de sistemas de irrigação por aspersão convencional.}

A Tabela 6 apresenta as diferenças na quantidade e dimensões de componentes nos sistemas de irrigação nas configurações " $\mathrm{A}$ " $\mathrm{e}$ "B" (FIGURA 1), quando dimensionados com: Vazão Econômica (VE) e Vazão Prática (VP).
Nas duas configurações ( $\mathrm{CA}$ e $\mathrm{CB}$ ) foram utilizados tubos com diâmetro de 4" na linha principal para VE, aumentando para 5" na VP. A principal diferença entre as configurações para a linha principal consistiu na quantidade de tubos, sendo maior na CA.

Por sua vez, a menor quantidade de tubos na linha principal na $\mathrm{CB}$ justificou-se pela existência de linhas secundárias, usadas com a função de distribuir a água às linhas laterais, não podendo serem chamadas de linha principal por não passar a vazão total por elas (Tabela 6).

Outra particularidade da CB foi a substituição do recalque, passando este a ser a linha principal, já que a vazão total passa apenas no suposto recalque. Já na CA o recalque existe e apresenta comprimento igual para VE e VP, diferindo apenas o diâmetro da tubulação, sendo maior na VP (Tabela 6).

Tabela 6. Quantidade de materiais nas configurações A e B.

\begin{tabular}{|c|c|c|c|c|c|}
\hline \multirow{2}{*}{ COMPONENTES DO SISTEMA } & \multirow{2}{*}{ UNIDADE } & \multicolumn{2}{|c|}{$\mathrm{CA}$} & \multicolumn{2}{|c|}{$\mathrm{CB}$} \\
\hline & & $\mathrm{VE}$ & VP & VE & VP \\
\hline \multicolumn{6}{|l|}{ LINHA PRINCIPAL } \\
\hline Tubulação aço zincado 4" & Tubos $6 \mathrm{~m}$ & 32 & - & 10 & - \\
\hline Tubulação aço zincado 5" & Tubos $6 \mathrm{~m}$ & - & 32 & - & 10 \\
\hline Válvula de derivações para ramais $3 ">2 "$ & Unid & - & - & 4 & - \\
\hline Válvula de derivações para ramais $3 ">3 "$ & Unid & - & - & - & 4 \\
\hline Válvula de derivações para ramais $4 ">3 "$ & Unid & 2 & - & - & - \\
\hline Válvula de derivações para ramais $5 ">3 "$ & Unid & - & 2 & - & - \\
\hline \multicolumn{6}{|c|}{ LINHA SECUNDÁRIA } \\
\hline Tubulação aço zincado 3" & Tubos $6 \mathrm{~m}$ & - & - & 53 & 53 \\
\hline \multicolumn{6}{|l|}{ LINHA LATERAL } \\
\hline Tubulação aço zincado 2" & Tubos $6 \mathrm{~m}$ & - & - & 78 & 0 \\
\hline Tubulação aço zincado 3" & Tubos $6 \mathrm{~m}$ & 82 & - & - & 78 \\
\hline Tubulação aço zincado 4" & Tubos $6 \mathrm{~m}$ & - & 82 & - & - \\
\hline Engate rápido com válvula automática para aspersos 1 " rosca & Unid & 28 & 28 & 28 & 28 \\
\hline Tubo de subida $1 \mathrm{~m}$ com 1 " dupla rosca & Unid & 28 & 28 & 28 & 28 \\
\hline Aspersor AGROPOLO. MV-360 & Unid & - & 28 & - & 28 \\
\hline Aspersor FABRIMAR ECO232 & Unid & 28 & - & 28 & - \\
\hline \multicolumn{6}{|l|}{ RECALQUE } \\
\hline Tubulação aço zincado 4" & Unid & 10 & - & - & - \\
\hline Tubulação aço zincado 5" & Unid & - & 10 & - & - \\
\hline
\end{tabular}

ACESSÓRIOS 


\begin{tabular}{cccccc}
\hline Chave compressora de 380V & Unid & 1 & 1 & 1 & 1 \\
Cotovelo aço zincado 90³" & Unid & - & - & 2 & 2 \\
Redução aço zincado 4" $>$ 3" & Unid & - & - & 1 & \\
Redução aço zincado 5" >3" & Unid & - & - & - & 1 \\
Registro de gaveta 3" & Unid & - & - & 2 & 2 \\
Registro de gaveta 4" & Unid & 1 & - & - & - \\
Registro de gaveta 5" & Unid & - & 1 & - & - \\
Tampão final 2" & Unid & - & - & 4 & - \\
Tampão final 3" & Unid & 2 & 2 & 1 & 5 \\
Tampão final 4" & Unid & 1 & 2 & - & - \\
Tampão final 5" & Unid & - & 1 & - & - \\
Tê aço zincado 90॰ 3" & Unid & - & - & 2 & 2 \\
Válvula de retenção 3" & Unid & - & - & 2 & 2 \\
Válvula de retenção 4" & Unid & 1 & - & - & - \\
Válvula de retenção 5" & Unid & - & 1 & - & - \\
\hline
\end{tabular}

CA - Configuração A; CB - Configuração B; VE - Vazão econômica; VP - Vazão prática.

Os diâmetros das linhas laterais diferiram com a modificação da vazão, para as duas configurações estudadas, como se ver na Tabela 6.

A modificação da Configuração acarretou no surgimento de novos componentes na CB, como: cotovelos de $90^{\circ}$, reduções e Tê (Tabela 6).
Na Tabela 7 tem-se a potência teórica e comercial do conjunto motobomba nas diversas situações de dimensionamento estudadas para a CA e CB. Vale salientar que a potência teórica foi inserida apenas para se entender a influência que os parâmetros estudados tiveram sobre o sistema de bombeamento.

Tabela 7. Potência teórica do conjunto Motobomba requerida de acordo com as configurações estudadas.

\begin{tabular}{ccccc}
\hline POTÊNCIA & \multicolumn{2}{c}{ CONFIGURAÇÃO A } & \multicolumn{2}{c}{ CONFIGURAÇÃO B } \\
$(\mathrm{Cv})$ & VE & VP & VE & VP \\
\hline Teórica & 22,10 & 24,70 & 25,61 & 27,67 \\
Comercial & 25,00 & 25,00 & 30,00 & 30,00 \\
\hline
\end{tabular}

VE - Vazão econômica; VP - Vazão prática.

Ainda de acordo com a Tabela 7 pode-se afirmar que o aumento da vazão influenciou no aumento da potência do sistema de bombeamento teórico em $11,76 \%$. No entanto, como no mercado não há disponibilidade de sistemas de bombeamento com variações menores de $5 \mathrm{Cv}$, foi necessário adquirir sistemas comerciais com a mesma potência.

Por sua vez, na CB a potência do sistema de bombeamento aumentou, tanto para VE quanto para $\mathrm{VP}$, motivado principalmente pela adição da perda de carga das linhas de derivação à altura manométrica. Tomando como base a influência da vazão constatou-se que para VP a potência teórica requerida foi maior. No entanto, a justificativa anterior para a potência comercial ser a mesma cabe nesta condição (Tabela 7).

Influência da vazão (VE e VP) no dimensionamento de sistemas de irrigação por aspersão convencional para CA e CB.

$\mathrm{Na}$ Tabela 8 estão representadas as principais características hidráulicas e de funcionamento do sistema quando dimensionado nas condições de diferentes vazões (VE e VP) para CA e CB. 
CARACTERIZAÇÃO DE SISTEMA DE IRRIGAÇÃO POR ASPERSÃO CONVENCIONAL DIMENSIONADO COM VAZÃO ECONÔMICA E PRÁTICA E DIFERENTES CONFIGURAÇÕES HIIDRÁULICAS

Tabela 8. Características do sistema de irrigação nas condições de diferentes vazões nas configurações A e B.

\begin{tabular}{|c|c|c|c|c|}
\hline \multirow{2}{*}{ CARACTERÍSTICAS DO SISTEMA } & \multicolumn{2}{|c|}{$\mathrm{CA}$} & \multicolumn{2}{|c|}{ CB } \\
\hline & VE & VP & VE & VP \\
\hline \multicolumn{5}{|c|}{ LINHA PRINCIPAL } \\
\hline Diâmetro teórico da LP $(\mathrm{mm})$ & 104,60 & 127,17 & 104,60 & 127,17 \\
\hline Diâmetro comercial interno da LP (mm) & 98,00 & 148,00 & 98,00 & 148,00 \\
\hline Comprimento da LP (m) & 180,00 & 180,00 & 50,00 & 50,00 \\
\hline Perda de carga na LP (mca) & 5,58 & 1,43 & 3,10 & 0,79 \\
\hline \multicolumn{5}{|c|}{ LINHA DE DERIVAÇÃO } \\
\hline Diâmetro teórico da LD (mm) & - & - & 73,96 & 63,58 \\
\hline Diâmetro comercial interno da LD (mm) & - & - & 73,00 & 73,00 \\
\hline Comprimento da LD (m) & - & - & 315,00 & 315,00 \\
\hline Perda de carga na LD (mca) & - & - & 16,52 & 12,83 \\
\hline \multicolumn{5}{|c|}{ LINHA LATERAL } \\
\hline Diâmetro teórico da LL (mm) & 73,03 & 85,47 & 49,21 & 57,59 \\
\hline Diâmetro comercial interno da LL (mm) & 73,00 & 98,00 & 48,00 & 73,00 \\
\hline Comprimento da LL (m) & 243,00 & 243,00 & 117,00 & 117,00 \\
\hline Perda de carga na LL (mca) & 6,29 & 3,14 & 6,49 & 1,75 \\
\hline Número de aspersores na LL & 14,00 & 14,00 & 7,00 & 7,00 \\
\hline Variação de pressão na LL (\%) & 17,48 & 9,73 & 17,96 & 5,59 \\
\hline \multicolumn{5}{|c|}{ ASPERSOR } \\
\hline Vazão do aspersor $\left(\mathrm{m}^{3} \mathrm{~h}^{-1}\right)$ & 2,20 & 3,27 & 2,20 & 3,27 \\
\hline Intensidade de aplicação $\left(\mathrm{mm} \mathrm{dia}^{-1}\right)$ & 6,80 & 10,30 & 6,80 & 10,30 \\
\hline Pressão de serviço utilizada (mca) & 30,26 & 28,89 & 30,26 & 28,89 \\
\hline \multicolumn{5}{|c|}{ DEMAIS INFORMAÇÕES } \\
\hline Tempo de irrigação por dia (h) & 10,00 & 10,00 & 10,00 & 10,00 \\
\hline Tempo para irrigar duas LL completas (h) & 3,71 & 2,50 & 3,71 & 2,50 \\
\hline Tempo ocioso (horas) & 0,00 & 13,31 & 0,00 & 13,31 \\
\hline Vazão horária do sistema $\left(\mathrm{m}^{3} \mathrm{~h}^{-1}\right)$ & 62,00 & 91,45 & 62,00 & 91,45 \\
\hline
\end{tabular}

VE - Vazão econômica; VP - Vazão prática; $\Delta \mathrm{P}$ - Variação de Pressão; LP - Linha Principal; LD - Linha de Derivação; LL - Linha Lateral

$\mathrm{Na}$ linha principal da CA observou-se a interferência do acréscimo da vazão (VP) principalmente no aumento do diâmetro e diminuição da perda de carga, mesmo sendo o comprimento igual para as duas situações (VE e VP). O aumento da vazão fez com que o diâmetro comercial interno da tubulação principal fosse maior $50 \mathrm{~mm}$. Com o aumento do diâmetro interno da tubulação houve diminuição de $74,38 \%$ da perda de carga. A justificativa dessa modificação é feita por Saad et al. (1994), onde descreveram que tubos de diâmetros menores são responsáveis por maior perda de carga por permitir mais atrito entre o tubo e a água, o que gera de forma relativa ao diâmetro um maior consumo de energia (Tabela 8).

O tipo de vazão também interferiu na perda de carga da linha lateral, diminuindo em $100,32 \%$ quando utilizou-se a vazão prática. Sabendo que a perda de carga sofre interferência direta do diâmetro da tubulação, comprimento da linha lateral e vazão, pode-se afirmar que este aumento decorreu estreitamente da vazão horária (aumento de $32,22 \%$ ), e do diâmetro teórico interno da tubulação. $\mathrm{O}$ diâmetro comercial da tubulação diferiu para VE e VP, sendo maior para a VP, já que esta apresenta vazão maior (Tabela 8). 
Sabe-se que no mercado não existem muitos diâmetros de tubo disponíveis, o que muitas vezes obriga ao projetista aumentar ou diminuir de forma drástica o diâmetro teórico para se encaixar no comercial, e assim ajustar o projeto aos critérios de dimensionamento (Tabela 8).

Quando a vazão econômica foi aumentada em $32,22 \%$ com o objetivo de tornar o sistema mais prático, houve modificação nas características hidráulicas do sistema, como: aumento da intensidade de aplicação do aspersor, necessitando de uma observação mais criteriosa na velocidade de infiltração básica de água no solo. Conforme Mantovani et al., (2009), intensidade de aplicação do aspersor deverá sempre ser menor que a velocidade de infiltração básica do solo (Tabela 8).

Ainda tomando como base a Tabela 8, desta feita para a CB, observa-se que a vazão econômica e prática permanece a mesma para o sistema nesta nova configuração. Por sua vez, a quantidade de aspersores na linha lateral é reduzida à metade, já que neste sistema o terreno é dividido por duas linhas de derivação, dando origem a linhas laterais com comprimento menor.

$\mathrm{Na}$ linha principal os diâmetros da tubulação foram respectivamente menores 50 $\mathrm{mm}$ para as duas vazões. Nesta situação a perda de carga assemelhou-se a CA, sendo a redução de $74,51 \%$ quando se utilizou a vazão prática.

Como já dito, nesta configuração a área foi dividida 2 (duas) porções iguais, sendo necessária a inserção de duas linhas de derivação para conduzir a água até as linhas laterais. Nas derivações as únicas características que apresentaram variações com o uso das diferentes vazões foram o diâmetro teórico e a perda de carga.

Algumas características das linhas laterais apresentaram similaridade quando na CA, como exemplo a perda de carga e a variação de pressão. No entanto, outras características diferiram, como o caso do diâmetro da tubulação que foi $25 \mathrm{~mm}$ menor para as duas vazões.
As demais características foram similares quando na $\mathrm{CA}$. Vale salientar que nesta situação de dimensionamento as linhas tidas antes como principais passam a ser secundárias, e o recalque passa a ser a linha principal.

\section{CONCLUSÕES}

A vazão prática (VP) proporcionou o manejo da irrigação mais eficiente, quando comparada com a vazão econômica (VE).

As características hidráulicas do sistema sofreram modificações com a alteração da vazão econômica (VE) para vazão prática (VP). Houve elevação do diâmetro da tubulação da linha principal e da linha lateral, como também aumento da perda de pressão na linha lateral e na linha principal. Foi notado esse comportamento para as duas configurações estudadas.

A modificação de configuração interferiu na variação da quantidade, dimensões e tipos de componentes do sistema. A configuração " $A$ " demandou tubulações com diâmetros maiores. Por sua vez, a configuração "B" demandou maior quantidade de tubulação, desta feita com diâmetros menores. Na configuração " $\mathrm{B}$ " ainda houve a necessidade da aquisição de novos componentes.

\section{AGRADECIMENTOS}

A Coordenação de Aperfeiçoamento de Pessoal de Nível Superior - CAPES, pela concessão da Bolsa do primeiro autor.

\section{REFERÊNCIAS}

BERNARDO, S.; SOARES, A. A.; MANTOVANI, E. C. Manual de irrigação. 8.ed. Viçosa: UFV, 2006. p. 625.

BIZARI, D. R.; MATSURA, E. E.; ROQUE, M. W.; SOUZA, A. L. Consumo de água e produção de grãos do feijoeiro irrigado em sistemas plantio direto e convencional. 
Ciência Rural, Santa Maria, v.39, n.7, p.20732079, out. 2009.

BRITO, R. R.; GRASSI FILHO, H.; SAAD, J. C. C.; OLIVEIRA, S. R. M. Produtividade do feijoeiro sob diferentes potenciais matriciais e fatores de depleção da água no solo. Nativa, Sinop, v. 03, n. 02, p. 109-114, abr/jun. 2015.

CENSO Agropecuário 1995-1996. Rio de Janeiro: IBGE 1998.

CENSO Agropecuário 2006. Brasil, grandes regiões e unidades da federação. Rio de Janeiro: IBGE, 2009. 777 p.

CUSTÓDIO, C. C.; VIVAN, M. R.; NUNES, R. C. A.; AGOSTIN, E. A. T. Tolerância cruzada induzida por choque térmico na germinação de semente de feijão. Revista Brasileira de Sementes, Londrina, v. 31, n. 1, p. 131-143, jan./mar. 2009.

DABACH, $\quad$ S.; $\quad$ SHANIA, U.; LAZAROVITCH, N. The influence of water uptake on matric head variability in a dripirrigated root zone. Soil and Tillage Research, Amsterdam, v.155, p.216- 224, Jan. 2016.

GONÇALVES, F. C.; MORAES, M. H. Porosidade e infiltração de água do solo sob diferentes sistemas de manejo. Irriga, Botucatu, v. 17, n. 3, p. 337 - 345, 2012.

KELLER, J.; BLIESNER, R. D. Sprinkle and trickle irrigation. New York: van Nostrand Reinold, 1990. 652 p.

MANTOVANI, E. C.; BERNARDO, S.; PALARETTI, L. F/. Irrigação: princípios e métodos. 3 eds. Viçosa, MG: UFV, 2009. 355 p.
PEREIRA, F. F. S.; PAI, E. D. MONTENEGRO, R. J. V.; ROMÁN, R. M.S.; GONZÁLEZ, A. M. G. O.; ESCOBEDO, J. F. Estudo comparativo da evapotranspiração de referência entre localidades no estado de São Paulo e na província de Habana. Irriga, Botucatu, v. 21, n. 2, p.395-408, maio/jun. 2016.

SAAD, J.C.C.; TOMAZELA, C.; PERES, J.G.; PERES, F.C.; FRIZZONE, J.A. Otimização da rede hidráulica de um sistema de irrigação por gotejamento utilizando programação linear. Pesquisa Agropecuária Brasileira, Brasília, v.29, n.5, p.797-805, /maio /1994.

SILVA, E. L.; PEREIRA, G. M.; CARVALHO, J. A.; VILELA, L. A. A.; FARIA, M. A. Manejo de irrigação das principais culturas. UFLA: FAEPE, 2000. $85 \mathrm{p}$.

SISTEMA Brasileiro de Classificação de Solos. 2 ed. Rio de Janeiro: Embrapa Solos, 2006. 306 p.

TURCO, J. E. P.; RIZZATTI, G. S.; PAVANI. L. C. Custo de energia elétrica em cultura do feijoeiro irrigado por pivô central, afetado pelo manejo da irrigação e sistemas de cultivo. Engenharia Agrícola, Jaboticabal, v.29, n.2, p.311-320, abr./jun 2009.

WUTKE, E. B.; ARRUDA, F. B.; FANCELLI, A. L.; PEREIRA, J. C. V. N. A.; SAKAI, E.; FUJIWARA, M.; AMBROSANO, G. M. B. Propriedades do solo e sistema radicular do feijoeiro irrigado em rotação de culturas. Revista Brasileira de Ciência do Solo, Viçosa, v.24, n.3, p. 621-633, jul./set. 2000 . 